\title{
Erratum to: Editoriale
}

\author{
Alessandro Masini \\ Direttore SC Ortopedia e Traumatologia, Ospedale Cristo Re, Roma, Italia \\ alemasini@mclink.it
}

Pubblicato online: 12 settembre 2014

(C) Springer-Verlag Italia 2014

\section{Erratum di: Lo Scalpello (2014) 28:73-74 \\ DOI 10.1007/s11639-014-0072-x}

Alessandro Masini, curatore del Fascicolo numero 2 di luglio 2014 de "Lo Scalpello" dal titolo "La gestione del paziente polifatturato", desidera fare una precisazione. A causa di una dimenticanza all'interno del suo Editoriale introduttivo sono stati omessi doverosi e importanti ringraziamenti ed è desiderio dunque del dr. Masini ringraziare ora il $\mathrm{dr}$. Federico Maria Sacchetti per l'attenta ed appassionata collaborazione nella revisione degli articoli inseriti all'interno della pubblicazione.

La versione online dell'articolo originale può essere trovata al doi:10.1007/s11639-014-0072-x. 